\title{
Disk evolution and the fate of water
}

\author{
Lee Hartmann • Fred Ciesla . \\ Oliver Gressel • Richard Alexander
}

Received: date / Accepted: date

\begin{abstract}
We review the general theoretical concepts and observational constraints on the distribution and evolution of water vapor and ice in protoplanetary disks, with a focus on the Solar System. Water is expected to freeze out at distances greater than 1-3 AU from solar-type central stars; more precise estimates are difficult to obtain due to uncertainties in the complex processes involved in disk evolution, including dust growth, settling, and radial drift, and the level of turbulence and viscous dissipation within disks. Interferometric observations are now providing constraints on the positions of $\mathrm{CO}$ snow lines, but extrapolation to the unresolved regions where water ice sublimates will require much better theoretical understanding of mass and angular momentum transport in disks as well as more refined comparison of observations with sophisticated disk models.
\end{abstract}

Keywords Accretion and accretion disks, · protoplanetary disks · atomic, molecular, chemical, and grain processes

\author{
L. Hartmann \\ Dept. of Astronomy, University of Michigan, Ann Arbor, MI USA \\ E-mail: lhartm@umich.edu \\ F. Ciesla \\ Dept. Geophysical Sciences, University of Chicago, Chicago, IL USA \\ E-mail: fciesla@uchicago.edu \\ O. Gressel \\ The Niels Bohr Institute, Blegdamsvej 17, 2100 Copenhagen Ø, Denmark
}

E-mail: gressel@nbi.ku.dk

R. Alexander

Dept. of Physics \& Astronomy, University of Leicester, University Road, LE1 7RH, UK

E-mail: richard.alexander@leicester.ac.uk 


\section{Introduction}

The first stages of planet formation begin within protoplanetary disks-the cloud of dust and gas that circle young stars during their first few million years of evolution. These disks form at the same time as their central stars, as a molecular cloud core collapses under the force of gravity. While much of the mass in the core eventually coalesces at the center to form a young star, the large amount of angular momentum in the core dictates that some of the mass settles into orbit around that star. It is within these disks that solid particles aggregate to form the seeds of planets and satellites, with the chemical compositions of these solids determined by the different environments that they are exposed to within the disk.

The physical and chemical conditions within protoplanetary disks evolve over millions of years as mass and angular momentum are transported during the final stages of their star's growth. Their structures are determined by a complex interplay between dust evolution, which affects the disk temperature, density and ionization distributions through absorption of light from the central star and any internal viscous heating, and transport processes which cause accretion, diffusion, and possibly turbulence (each of which affect the dust distribution). In particular, the specific locations of water ice and vapor within the disk, and therefore the implications for the possible delivery of water to terrestrial planets, remain uncertain due to uncertainties in transport, viscous heating, and dust growth and settling, as we discuss in this review.

\subsection{Protoplanetary Disk Basics}

The standard reference point in discussing the structure of protoplanetary disks is the Minimum Mass Solar Nebula (Weidenschilling 1977; Hayashi 1981). The idea for this hypothetical disk came from trying to reconstruct the solar nebula by taking the mass of planets in our Solar System and distributing it in a series of "feeding zones" for each planet to recover a smooth distribution. Hydrogen gas was then added to recover a solar mix of elements (dust-to-gas ratio of $\sim 0.01$ when water ice was condensed and $\sim 0.005$ when water remained as a vapor). The total surface density (mass per unit area in a given annulus) of such a disk is given by:

$$
\Sigma(r)=1700\left(\frac{r}{1 \mathrm{AU}}\right)^{-\frac{3}{2}} \mathrm{~g} \mathrm{~cm}^{-2} .
$$

This gives a total disk mass out to $100 \mathrm{AU}$ of $\sim 0.01 M_{\odot}$. The temperature structure was found by determining the directly-irradiated blackbody temperature as a function of distance from the Sun (Hayashi 1981):

$$
T(r)=280\left(\frac{r}{1 \mathrm{AU}}\right)^{-\frac{1}{2}} \mathrm{~K}
$$



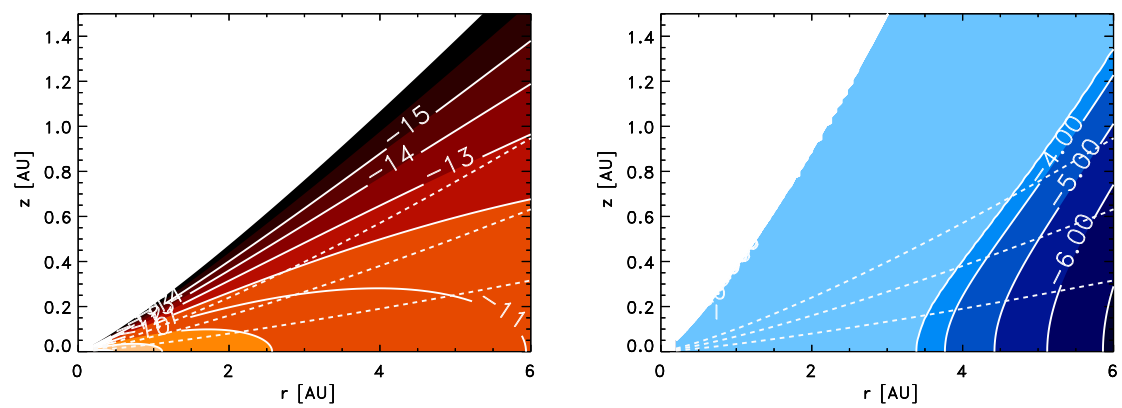

Fig. 1 Left: Two-dimensional density structure of the inner regions of the Minimum Mass Solar Nebula as described in the text. Contours represent $\log _{10}$ of the total gas mass density. The dashed lines represent the heights of 1,2 , and 3 scale heights within the disk. Right: Two-dimensional distribution of water vapor in the disk. Canonical $\mathrm{H}_{2} \mathrm{O} / \mathrm{H}_{2}$ number ratios are $5 \times 10^{-4}$, which are found inwards of the midplane snow line (just inward of $3.5 \mathrm{AU}$ here). Contours are $\log _{10}$ of the $\mathrm{H}_{2} \mathrm{O} / \mathrm{H}_{2}$ ratio.

It is not clear how accurately the above structure reflects the properties of the solar nebula. For example, there are significant uncertainties in the temperature structures of protoplanetary disks because they are optically thick, at least to the star's radiation and in parts to their own internal radiation field, and viscous dissipation may serve as an additional source of heating, so that the application of equation (2) is questionable (see discussion in 1.4). As we discuss further below, disks are also evolving due to a variety of processes, transporting mass inward to be accreted by the central star or losing mass due to photoevaporative radiation; thus the surface density and temperature are unlikely to be constant in time. Moreover, the mass of the MMSN is a minimum by definition, and other models suggest higher surface densities (Desch 2007).

Despite these issues, the MMSN serves as a reference model to illustrate key properties and structures by which we can understand real protoplanetary disks. Proceeding further, one must consider the vertical structure of the disk, assumed to be set by hydrostatic equilibrium as follows:

$$
\rho_{g}(z)=\frac{\Sigma}{(2 \pi)^{1 / 2} h_{g}} \exp \left(-\frac{z^{2}}{2 h_{g}^{2}}\right)
$$

where $\rho_{g}$ is the $z$ is the height above/below the disk midplane and $h_{g}$ is the disk scale-height, with $h_{g}=c_{s} / \Omega$, where $c_{s}$ is the local speed of sound and $\Omega$ the local Keplerian frequency. For simplicity, the disk is assumed to be vertically isothermal, allowing $c_{s}$ and $h_{g}$ to be constant with height. Figure 1 shows the plot of the gas density structure for the MMSN around the terrestrial planet region of the Solar System. In the absence of any dust dynamics (see below), small dust grains will be suspended within the disk, at least initially, determined largely by whether water is condensed or not as described above. 


\subsection{Snow Line}

A key element in the discussion of water is the location of the snow line (SL) in the disk; that is the distance from the star where water begins to freeze-out and become a solid. Any water vapor that moves outward from this location condenses, while any water ice that migrates inward evaporates, and thus this location is also referred to as the water ice condensation/evaporation front. This location can be determined from both equilibrium and kinetic arguments. In the equilibrium approach, the snow line is set based on thermodynamic data; that is, it is the location in the disk where, if all water was present as a vapor in the disk, its partial pressure would exceed the equilibrium vapor pressure at the local temperature.

An alternative approach defines the location of the snow line based on chemical kinetics, which is useful as thermodynamic equilibrium is not always achieved in a protoplanetary disk. In this manner, the snow line can be determined by finding where the freeze-out (adsorption) flux of water molecules onto grains equals the desorption flux of molecules off of grain surfaces. Following Bergin et al. (2013), the two fluxes can be expressed as:

$$
\begin{gathered}
F_{\mathrm{ads}}=0.25 n_{\mathrm{H}_{2} \mathrm{O}} v_{t h}, \\
F_{\mathrm{des}}=\nu_{0} \exp \left(-E_{b} / k T\right) N_{s},
\end{gathered}
$$

where $n_{H_{2} O}$ is the local number density of gaseous water molecules, $v_{t h}$ is the thermal velocity of those water molecules, $E_{b}$ is the experimentally determined binding energy of water onto a grain surface, $N_{s}$ is the density of adsorption sites on the surface of a grain $\left(\sim 10^{15} \mathrm{~cm}^{-2}\right)$, and $\nu_{0}$ is a vibrational frequency of order $10^{12} \mathrm{~s}^{-1}$. In instances where $F_{\text {des }}>F_{\text {ads }}$, water remains completely in the vapor phase. When $F_{\text {ads }}>F_{\text {des }}$, water molecules begin to freeze-out onto dust grains until $n_{H_{2} O}$ drops to a value such that the two fluxes are equal. Taking the water (vapor+ice) to hydrogen ratio throughout the disk as a constant $n_{H_{2} \mathrm{O}}^{\mathrm{Tot}} / n_{\mathrm{H}_{2}} \sim 5 \times 10^{-4}$ (Lodders 2003. Cleeves et al. 2014) and typical protoplanetary disk midplane $(z=0)$ conditions, the snow line will generally occur at temperatures of $T \sim 120-170 \mathrm{~K}$. These temperatures are in general agreement with those predicted from equilibrium studies (e.g. Lodders 2003).

The snow line is often pictured as being a singular radial location in the disk, $R_{\mathrm{SL}}$, the point at the disk midplane where $F_{\text {ads }}=F_{\text {des }}$ can only be maintained if some of the water in the vapor phase is removed and put onto solids. However, due to the decrease in gas density with height above the disk midplane, the snow line actually has a vertical structure. If the disk is vertically isothermal, $F_{\text {ads }}$ will decrease exponentially with height (following the background gas density as assumed in Eq. 3), while the desorption flux will remain constant. Thus, the snow line will begin at the disk midplane at $R_{\mathrm{SL}}$, with only a small fraction of water being incorporated into solids and the rest remaining as vapor with height. As seen in Fig 1 . for $r>R_{\mathrm{SL}}$, the amount of water in the disk present as ice increases; this is due both to the increasing height of the snow line and the drop of temperatures leading to more water being removed from the gas phase. 


\subsection{Dust and Gas Dynamics in Protoplanetary Disks}

Upon formation of a protoplanetary disk, the dust grains and gas are generally thought to be uniformly mixed, with $\sim 99 \%$ of the mass found in gas and $\sim 1 \%$ as condensed solid grains ranging from submicron to microns in size (Lodders 2003). Over time, the solids and gas will be subjected to a series of dynamic processes which will lead to them evolving differently from one another and aid in the formation of planetary building blocks.

While the vertical structure of the gas in a protoplanetary disk is determined by the hydrostatic balance between the component of stellar gravity that pulls material towards the disk midplane with the pressure of the gas, solids feel less pressure support and will settle through the gas toward the midplane. Larger solids settle more rapidly than fine dust. Turbulence, if present, will work to offset the growing concentration of solids at the disk midplane, lofting materials to higher atltitudes again. The extent to which solids of a given radius, $a$, settle in a disk is determined by the dimensionless stopping time of the particle:

$$
\tau_{s}=\frac{\rho a}{\rho_{g} c_{s}} \Omega
$$

where $\rho$ is the material density of the solid. (This quantity is also referred to as the Stokes number in the literature. e.g. Cuzzi et al. 1993). The amount of turbulence in a disk is uncertain, as described below. Models typically quantify it by defining a dimensionless parameter, $\alpha$ which produces an effective viscosity, $\nu=\alpha c_{s} h_{g}$. This turbulence would also produce a diffusivity within the gas which is often taken as $D$ as being of order $\nu$, though differences in magnitude are possible. If we assume $D=\nu$, for the particles we are interested in, then those with $\tau_{s} / \alpha<<1$ will remain suspended throughout the height of the disk (giving them a dust layer thickness of $h_{d} \sim h_{g}$ ), while larger particles will settle into a dust layer with thickness (Youdin and Lithwick 2007):

$$
h_{d}=\left[1+\frac{\tau_{s}}{\alpha}\left(\frac{1+2 \tau_{s}}{1+\tau_{s}}\right)\right]^{-\frac{1}{2}} h_{g} .
$$

It is worth noting that larger particles, as they decouple from the gas, will have effective diffusivities less than that of the gas. Turbulence may also redistribute materials radially in the disk, but this is only important for gases and small solids (those small enough to remain coupled to the gas with $\tau_{s}<\alpha$ ).

Larger particles will also begin to decouple from the gas through their radial motions in the disk. While the gas in the disk orbits its central star, its velocity is usually slightly less than Keplerian due to the generally outward pressure gradient (hot, dense gas near the star; cool, sparse gas further out). Thus, while the star exerts a gravitational force on the gas, the net central force is somewhat less due to the pressure support it experiences. Solid particles get negligible support from this pressure gradient, and thus would otherwise orbit the star at Keplerian rates. As a result, the solids experience a headwind in their orbits around the star. Solids with $\tau_{s} \ll 1$ continue to remain coupled 
to the gas and are minimally affected by aerodynamic drag, while those with $\tau_{s} \gg 1$ have so much inertia that the headwind can largely be ignored. However, those particles with $\tau_{s} \sim 0.01-10$ (typically $\mathrm{mm}$ to meter sizes) drift inwards as a result of the loss of energy and angular momentum arising due to the headwind.

The collective effect of these dynamical mechanisms results in a complex evolution of the dust-to-gas ratio as a function of time, with important but uncertain consequences for the building of large bodies and the global water distribution, as discussed next.

\subsection{Viscous heating and radiative transfer effects}

The simple optically-thin equation (2) for the disk temperature is unrealistic because protoplanetary disks are optically thick to radiation from the central star. Beyond a few AU or so, temperatures are dominated by irradiation from the central star. The precise heating rate is dominated by the "flaring" of the disk (i.e., the layers which absorb the starlight lie at heights $z / R \propto R^{\eta}$, $\eta>0$; Kenyon and Hartmann (1987)). In the case of dust particles with properties similar to that of the interstellar medium (ISM) that are well-mixed with the gas, the simple model of Chiang and Goldreich (1997) for opticallythick disk absorption of starlight yields an effective (disk surface) temperature distribution $T_{e, r} \propto R^{-3 / 7}$, while more detailed models yield a slightly steeper dependence on radius,

$$
T_{e, r} \approx 220 L_{1}^{1 / 4} R_{A U}^{-1 / 2} \mathrm{~K}
$$

(D'Alessio et al. 1998, 2001), where the stellar luminosity is in units of solar. However, the midplane temperature for such optically-thick disks is considerably lower, often by a factor of two at a few AU. It appears that irradiation heating by itself is not able to move the water snow line significantly out beyond $1 \mathrm{AU}$, which poses problems for understanding how chondritic meteorite parent bodies, thought to form outside of $\sim 1$ AU late in solar nebula evolution, are water-poor (e.g. Abe et al. 2000).

The apparent limitations of stellar irradiation for explaining the variation of water abundances in the solar system has led to proposals that accretion heating is the dominant factor setting the disk central temperature (e.g., Garaud and Lin 2007, Kennedy and Kenyon 2008, Bitsch et al.|2015). To fix ideas, consider a toy model of a optically-thick, steady, viscously accreting disk, with effective temperature of

$$
T_{e, a}=\left(\frac{3 G M \dot{M}}{8 \pi \sigma R^{3}}\right)^{\frac{1}{4}} \sim 85 M_{1} \dot{M}_{-8} R_{A U}^{-3 / 4} \mathrm{~K},
$$

where we have assumed fiducial values of a central star of one solar mass and an accretion rate of $10^{-8} M_{\odot} \mathrm{yr}^{-1}$ as typically observed. If the disk is very optically thick and the viscous dissipation occurs near the midplane, radiative 
trapping can increase the central temperature $T_{c, a}$ over $T_{e, a}$ by a factor of $\sim(3 \tau / 8)^{1 / 4}$ Hubeny 1990), where $\tau$ is the vertical Rosseland mean optical depth. The optical depth is $\tau=k_{R} \Sigma / 2$, where $k_{R}$ is the Rosseland mean opacity and $\Sigma$ is the surface density of the disk, which is related to the mass accretion rate $\dot{M}$ in the standard (vertically-isothermal) viscous disk as

$$
\dot{M}=3 \pi \alpha c_{s}^{2} \Sigma \Omega^{-1},
$$

bf where $\alpha$ is the usual parameterization of the viscosity (see 33 ) and $c_{s}$ is the sound speed. The result is

$$
T_{c, a}=\left[\frac{3}{64 \pi^{2}} \frac{G M \dot{M}^{2}}{\sigma R^{3}} \frac{\mu \Omega k_{R}}{\alpha R_{g}}\right]^{1 / 5}=330 M_{1}^{3 / 10} \dot{M}_{-8}^{2 / 5} k_{R}^{1 / 5} \alpha_{-3}^{-1 / 5} R_{A U}^{-9 / 10} \mathrm{~K},
$$

where $\alpha_{-3}$ is in units of $10^{-3}$ and $R_{g}$ is the gas constant, and for simplicity we have ignored any temperature or density dependence of $k_{R}$.

Assuming ice condenses at temperatures 170-120 K, and setting $k_{R} \sim$ $1 \mathrm{~cm}^{2} \mathrm{~g}^{-1}$, the crude estimate of equation 11 indicates a snow line at $\sim 2.1-$ 3.1 AU for the fiducial parameters, is reasonably consistent with more detailed calculations (e.g., Bitsch et al. 2015, see Figure 2). However, this estimate may well be an upper limit to the increase in temperature due to viscous heating. As discussed in $\$ 1.3$, dust evolution is likely to reduce the optical depth, as growth beyond the ISM grain size distribution will have lower opacities. Emprical estimates suggest depletion of small dust in the upper disk layers by factors of $10^{-1}$ to $10^{-3}$ (§2.1, Furlan et al. 2006); presumably this is the result of growth as the large particles settle to the midplane (as needed to explain mm emission; D'Alessio et al. 2001)). The lower panels of Figure 2 show that the snow line moves in to $R \sim 1 \mathrm{AU}$ for a depletion of $10^{-2}$ of small dust in the upper layers. Theory suggests that such depletion should occur rapidly (depending upon the level of turbulence present), so the assumption of no depletion seems problematic.

Higher accretion rates and fragmentation to replenish small dust grains can help compensate for reduced trapping of heat by dust depletion (e.g., Bitsch et al. 2015). However, mass accretion rates drop significantly with time, on timescales of 3 Myr or less (2.2. Hartmann et al. (2016)) and dust growth even with fragmentation is rapid (2.1). Morever, it is not clear that viscous dissipation occurs mostly near the midplane, or even that viscosity is important in transporting angular momentum in large regions of the disk, making its importance as a heat source questionable (see \$3).

Major outbursts of rapid accretion - up to $10^{-4} M_{\odot} \mathrm{yr}^{-1}-$ are known to occur in some young objects (the FU Ori variables; Hartmann and Kenyon 1996), whose disks are demonstrably internally-heated and thus viscous-dissipation dominated. Such high accretion rates can move snow lines farther out (Cieza et al. 2016), but these large outbursts are confined to early evolutionary phases (ages < $1 \mathrm{Myr}$ ); any remnants of these episodes may well be accreted into the star by the time of planetesimal formation. 

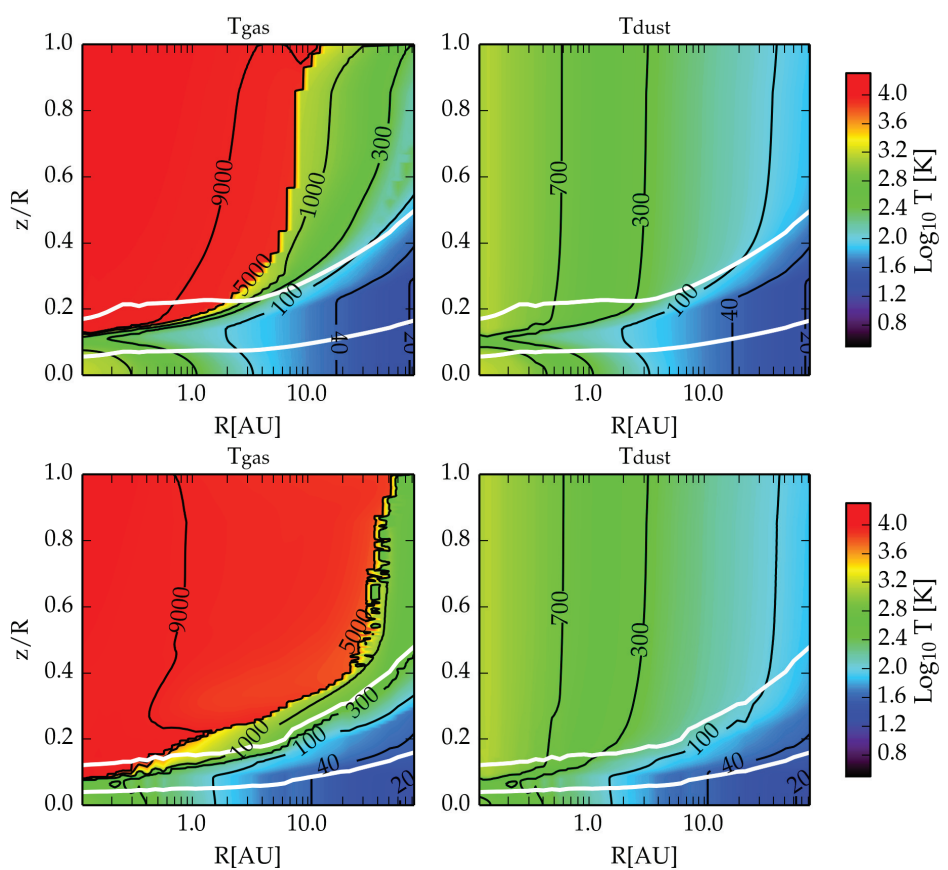

Fig. 2 Upper panels: gas (left) and dust (right) temperatures for a detailed radiative transfer model with viscous heating with $\dot{M}=10^{-8} M_{\odot} \mathrm{yr}^{-1}, \alpha=10^{-3}$. The SL at the midplane lies at $R \sim 2-3 \mathrm{AU}$, in agreement with equation 11 for the toy model. Lower panels: the same global model properties, but now assuming that small dust is depleted by a factor of 100 in the upper layers. Now the SL moves inward to $\sim 1 \mathrm{AU}$, due to the decreased trapping of the viscously-generated radiation. Courtesy Nuria Calvet and Ramiro Franco Hernandez.

\section{Observational constraints on disk structure and evolution}

The density and internal temperature structure of protoplanetary disks are not well constrained. Most of our direct information is limited to scales of several to to hundreds of AU. The inner disk structure near the expected snow line at a few AU (for a solar-luminosity star) is not easily resolvable with current techniques, and there are indications that inner disks are optically thick (due to dust) at mm wavelengths. One therefore must work to connect models and observations on larger scales to attempt any extrapolation to the water SL.

\subsection{Dust}

Most of what we infer about the radial and vertical temperature and density distributions of disks comes from observations of dust emission, as cold molecular hydrogen is not detectable and most other molecular tracers are either optically-thick, are activated only in disk upper layers, or are subject to uncertainties in chemical reaction rates. As shown in Figure 3, we infer that the dust in disks around solar-mass pre-main sequence ( $\mathrm{T}$ Tauri) stars exhibit a sort 


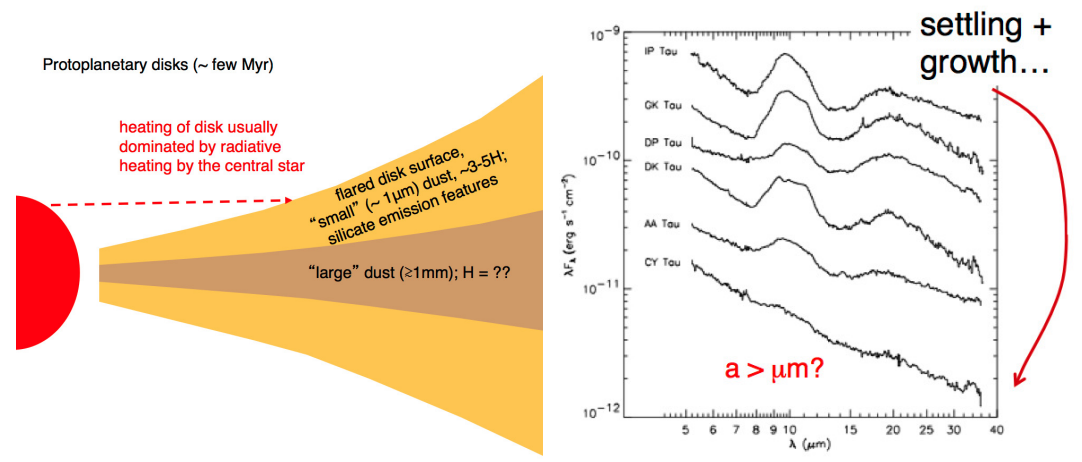

Fig. 3 The left panel schematically indicates structure of solar-mass pre-main sequence (T Tauri) disks. Small dust must be mixed with the gas to at least a few scale heights to explain the observed infrared dust emission (right panel), both in terms of a continuum and also to explain the silicate emission feature at $\lambda \sim 10 \mu \mathrm{m}$. Large $(\mathrm{mm})$ dust must be present to explain the long-wavelength emission; presumably this dust resides in a relatively thin layer at the disk midplane. The set of mid-infrared spectral energy distributions in the right panel, taken from Furlan et al. (2006), is suggestive of a sequence of increased settling of dust, making the infrared excess fall off faster to longer wavelengths, along with a decrease in the strength of the silicate emission feature which may indicate grain growth. Note that while some stars exhibit emission consistent with amorphous silicates (e.g., IP Tau), others clearly show the presence of crystalline silicate particles (e.g., DK Tau).

of "two layer" structure, with large dust concentrated near the disk midplane and small dust suspended in upper layers to a few scale heights. The $10-20 \mu \mathrm{m}$ silicate emission features seen in most $\mathrm{T}$ Tauri disks can be explained by dust with sizes smaller than a few $\mu \mathrm{m}$, while the amount of mm-wave emission is only explainable with reasonable dust masses if the grains have sizes of order $1 \mathrm{~mm}$ or larger (e.g., D'Alessio et al. 2001). Placing the small dust in upper layers where the vertical temperature inversion (caused by irradiation due to the central star) results in emission features, while distributing the large dust closer to or in the midplane avoids the elimination of silicate features.

As dust grows and settles to the midplane (see 1.3 , small dust becomes depleted in the upper layers. Indeed, theoretical models of this process in the absence of significant turbulence suggest this should happen quickly, posing challenges to explain the observed existence of suspended small dust in disks of ages as small as $\sim 1 \mathrm{Myr}$ (Dullemond and Dominik|2005; Birnstiel et al.|2011). Empirical estimates of small dust depletion in upper disk layers are difficult to make with any accuracy, but current models to explain observations suggest depletions by factors of 10 to 1000 (D'Alessio et al. 2001; Furlan et al. 2006), with significant implications for radiative trapping of viscous heat (\$1.4).

Efforts to demonstrate grain growth generally are based on observations of spectral indices in the mm-wave region. Small ISM dust has a steep dependence of opacity on frequency $k_{\nu} \propto \nu^{-\beta}$, with $\beta \sim 1.7$, as a consequence of the limited sizes of the largest grains $(\sim 0.3 \mu)$. Observations of mm-wave and $\mathrm{cm}$-wave emission from disks at distances of $\sim 20-100$ AU indicate much smaller 
values of $\beta \sim 1$ or even 0 , indicating growth to at least $\mathrm{mm}$ or even $\mathrm{cm}$ sizes (Pérez et al. 2012, 2015, Tazzari et al. 2015). Unfortunately, maximum particle sizes cannot be determined once dust grows in size beyond the wavelengths of observation; in this case $\beta$ is determined by the shape of the particle size distribution. As emission at $\mathrm{cm}$ wavelengths is generally quite weak in outer disks (50-100 AU), currently it is not possible say anything about $\gtrsim \mathrm{cm}$-sized dust at large radii.

Given the uncertainties in dust (and solid) size distributions, dust disk masses must be considered to be lower limits; that is, what is being measured at $\mathrm{mm}$ wavelengths is only the amount of mass in solids of sizes $\sim 100 \mu \mathrm{m}$ to $\sim$ a few mm. The survey of (Andrews and Williams 2005) yielded a median "disk mass" (100 $\mathrm{x}$ the solid mass) of $\sim 0.005 M_{\odot}$. Statistical estimates from gas accretion yield somewhat larger values $(2.2)$.

Another complicating effect in addition to vertical settling is radial drift. This effect has been seen in a number of objects, most clearly in the nearby T Tauri star TW Hya with mm-dust grains having a sharp edge at 60 AU, while CO gas emission is seen out to $\sim 200$ AU (Andrews et al. 2012). As drift of $\mathrm{mm}-\mathrm{cm}$ sized particles is quite rapid, this causes some problems in understanding the existence of dust in this size range out at $\gtrsim 50 \mathrm{AU}$, particularly in this relatively old object (age $\sim 10 \mathrm{Myr}$ ).

\subsection{Gas}

The only current constraint on the mass of molecular hydrogen in disks is the estimate of $M_{d} \sim 0.06 M_{\odot}$ from analysis of HD in TW Hya (Bergin et al. 2013). Even this result is model-dependent, as it relies both on the assumed isotopic ratio and, more importantly, the disk temperature structure, as HD does not probe the coldest regions where most of the mass resides.

Williams and Best (2014) attempted to use rotational emission lines of ${ }^{12} \mathrm{CO},{ }^{13} \mathrm{CO}$, and $\mathrm{C}^{18} \mathrm{O}$ isotopologues to correct for optical depth effects and variations in gas temperatures as a function of radius and height. Assuming a conversion of $\mathrm{CO}$ to $\mathrm{H}_{2}$, they derived gas masses of order $10^{-3} M_{\odot}$, an order of magnitude smaller than expected from dust masses (times 100). More importantly, such gas masses are also an order of magnitude too small to sustain typical accretion rates of $10^{-8} M_{\odot} \mathrm{yr}^{-1}$ over timescales of 1-3 Myr (see below). One possible resolution is that $\mathrm{CO}$ freezes out onto grains, removing it from the gas phase and thus rendering it undetectable in mm-wave rotational line emission.

Gas mass accretion rates $(\dot{M})$ are important in providing statistical upper limits to the possible viscous heating in disks. $\dot{M}$ increases with increased stellar mass and decay with age. The best fit for $\mathrm{T}$ Tauri stars with a median age of about 1-2 Myr derived by G. Herczeg is

$$
\log \left(\dot{M} / M_{\odot} \mathrm{yr}^{-1}\right) \approx-7.9+2.1 \times \log \left(M_{*} / M_{\odot}\right)
$$




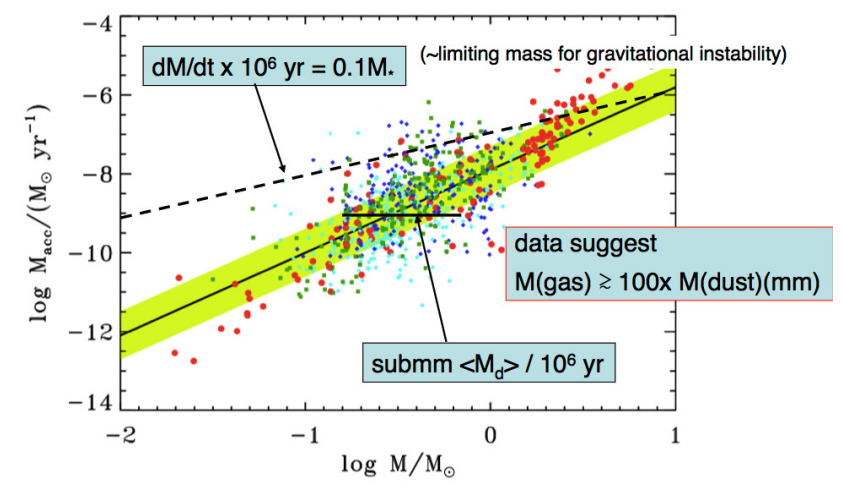

Fig. 4 Observationally estimated accretion rates of T Tauri stars at typical ages $\sim 1-2 \mathrm{Myr}$ as a function of stellar mass. There is a clear trend of increased mass accretion with increasing stellar mass, though there is substantial scatter. The dashed line indicates the rate that would result in accreting $10 \%$ of the stellar mass from the disk; it seems unlikely that disks can be considerably more massive than this, as they would be gravitationally unstable. (Stars with masses $>1 M_{\odot}$ appear to have shorter average disk lifetimes.) The data suggest that $\mathrm{T}$ Tauri stars typically accrete a mass equal to the inferred dust masses, suggesting that the mm-wave emission analysis generally underestimates the gas mass. Modified from Hartmann et al. (2016).

(see Hartmann et al. 2016). As one can see from Figure 4 , there is a large scatter at any mass, more than the factor $\sim 0.5$ dex expected for observational errors. Accretion rates also generally decrease with increasing age (Figure 5), although the rates of decay are uncertain, due in part to difficulties in assigning ages to individual young stars (Soderblom et al. 2014). This decrease in $\dot{M}$ with time, along with the likely depletion of small dust with increasing age, indicates that the snow line should move inward during the main optically thick disk lifetime (\$1.4).

The "lifetimes" of disks as judged from either infrared dust emission (left panel of Figure 5) or from the ending of detectable accretion onto the central star (Fedele et al. 2010), exhibit a significant spread. The "usual" disk lifetime is often given as $3 \mathrm{Myr}$, but a few (optically-thick) disks survive to $\sim 10 \mathrm{Myr}$, while others last for $\lesssim 2$ Myr. It appears that both dust emission and accretion disappear fairly rapidly (see below), so there must be something like a two-phase behavior, with a moderate decay lasting for 1-10 Myr and a rapid decrease over $<1$ Myr. This rapid clearing could be produced by photoevaporative mass loss from the disk driven by X-ray or other high-energy radiation (Alexander et al. 2006, Owen et al. 2011), or by giant planet formation.

\subsection{Gaps and holes}

The advent of sensitive, high-spatial resolution mm-wave interferometry, especially using the Atacama Large Millimeter and Submillimeter Array (ALMA), has revealed that major radial structuring of protoplanetary disks is far more 

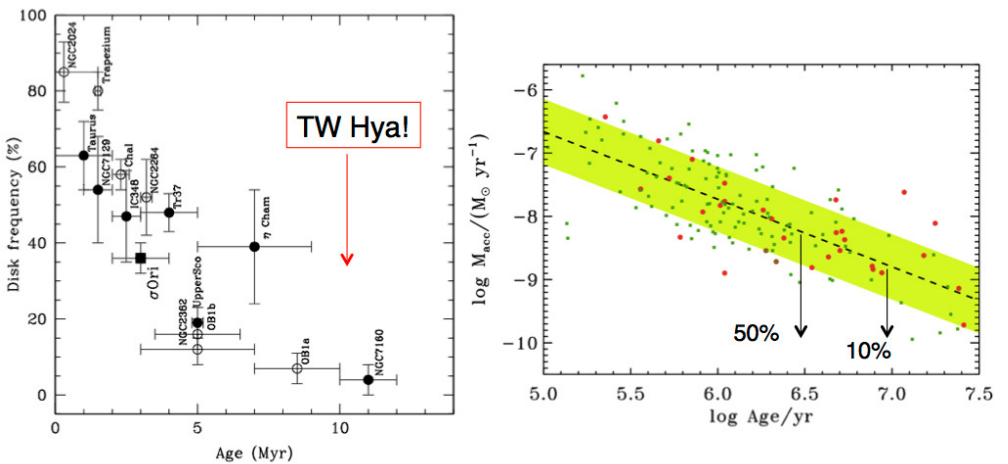

Fig. 5 Left: frequency of detectable infrared dust disk emission in the IRAC bands $3.5-8 \mu \mathrm{m}$ as a function of group or cluster age. From Hernández et al. (2008). Right: dependence of mass accretion rates on age, scaled to account for the mass dependence of equation 12 for stars with masses in the range 0.3-1.0 $M_{\odot}$ (see also Fig. 4). The mass-accretion rate relationship is calculated for objects with mass between $0.05-1 \mathrm{M}_{\odot}$. The resulting fit is uncertain due to correlated errors (see text). The arrows emphasize that significant numbers of accreting disks "drop out" with increasing age. From Hartmann et al. (2016).

common than previously realized. Disks of substantial masses but with inner holes strongly depleted in small dust have been increasingly recognized (the "transitional disks"; Calvet et al. 2002, Espaillat et al. 2014, Figure 6). In addition, there are also disks with inner disk dust as well as a large gap (the "pre-transitional disks"; Espaillat et al. 2007). Recent observations suggest that the gas within these holes and gaps (Bergin et al. 2003, Najita et al. 2007; Ingleby et al. 2009) is also depleted, although by smaller factors than of the dust (van der Marel et al. 2016). Because observations still generally detect only the largest holes and gaps, the new results suggest that there may not be any truly "primordial" or "protoplanetary" disks once the infall of envelope material to the disk during the protostellar phase has ended. Significant formation of planetesimals and embryos, if not planetary cores, probably begins very early, $\lesssim 1 \mathrm{Myr}$, especially if giant planets are responsible for creating large disk holes and gaps. This is consistent with evidence from the Solar System; rapid formation of planetesimals is supported by radiometric dating of iron meteorites, which suggest planetesimals formed in our solar nebula concurrently or within $10^{5}$ years of the formation of CAIs, the oldest objects in our Solar System, and which define $t=0$ in cosmochemical clocks (Kruijer et al. 2014). Such bodies would predate the accretion of chondritic meteorite parent bodies by 2-3 Myr (Connelly et al. 2012).

An interesting sidelight to the presence of gaps and holes strongly cleared of small dust in young disks is the possibility that even rapid planetesimal and embryo formation might occur in optically-thin regions (see \$5). 

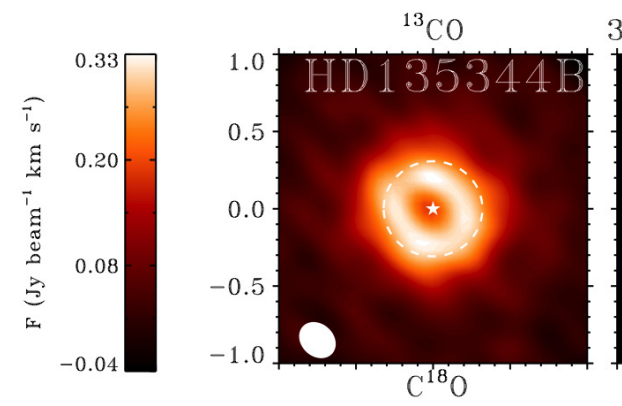

$345 \mathrm{GHz}$ continuum

Fig. 6 ALMA imaging of the $1.6 M_{\odot}, 8 L_{\odot}$ young star HD $135344 \mathrm{~B}$, showing that gas emission $\left(\mathrm{C}^{18} \mathrm{O}\right)$ is present inside the dust disk as seen in mm-wave emission. Similar structures are seen in several other systems, suggesting that planet formation has trapped large dust particles in the outer disk while allowing some gas to move inward, helping to explain the observed gas accretion onto central stars (e.g., Ingleby et al. 2014). The non-axisymmetric structure in the dust may indicate a vortex, suggesting perturbations by planets. From van der Marel et al. (2016).

\subsection{Observations of Water in Protoplanetary Disks}

Water vapor has been detected in several $\mathrm{T}$ Tauri and Herbig Ae/Be stars (Carr and Najita 2011; Brittain et al. 2015, Blevins et al. 2015). $\mathrm{H}_{2} \mathrm{O}$ emission lines are seen superposed on the strong dust continuum, showing that they arise in upper layers of the disk, with typical gas temperatures $\sim 600 \mathrm{~K}$ (Carr and Najita 2011). Unfortunately, translating these observations of surface water vapor, estimated to originate at radii $\sim 0.5-5 \mathrm{AU}$, into midplane snowline positions is highly model-dependent (Blevins et al. 2015), particularly because the high temperatures of the upper disk gas layers tend to reflect heating by stellar X-rays and ultraviolet radiation (Glassgold et al. 2009).

Detection of where water starts to freeze out onto grains has proven to be more challenging. Even with the vast improvements made by the ALMA interferometry, spatial resolution is limited to a few AU in the nearest star-forming regions, and to the brightest objects. As a check on our general understanding of disk temperature structures, $\mathrm{CO}$ snow lines have been detected indirectly at scales of tens to nearly $100 \mathrm{AU}$ (Qi et al. 2013), depending upon the luminosity of the central star. CO was not directly observed; instead, the inner edge of emission from $\mathrm{N}_{2} \mathrm{H}^{+}$was taken to be the position of the $\mathrm{CO}$ snow line because this ion is only abundant in regions where $\mathrm{CO}$ is depleted from the gas. Similar tracers do not exist for $\mathrm{H}_{2} \mathrm{O}$, making detection of the water snow line a challenge. Further, the likely positions of water snow lines in the nearest protoplanetary disks are at the edge or below current imaging capabilities. Banzatti et al. (2015) suggested that the water snow line might be inferred from a change in the spectral index due to the decrease in dust size and optical properties as water ice is sublimated, but the potentially optically-thick nature of the inner disks may make these observations difficult. Crystalline water ice has been detected in a few disks from spatially-unresolved observations (Mc- 

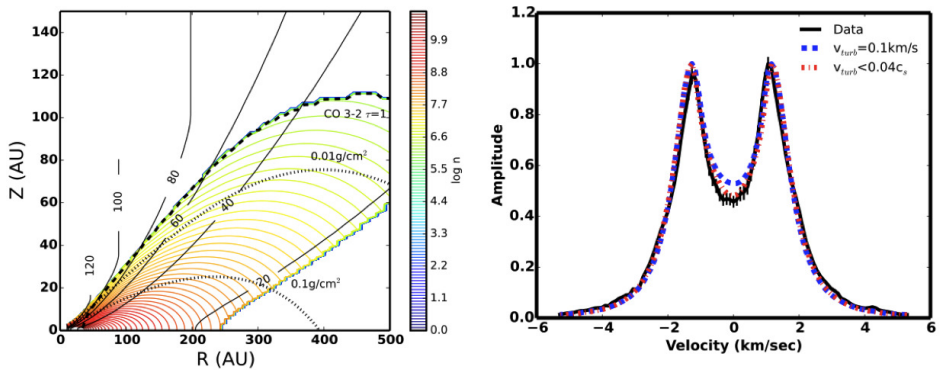

Fig. 7 Left: disk model constructed to explain the CO 3-2 emission from the disk of HD 163296. The black solid curves denote isotherms in $\mathrm{K}$, the dotted curves show contours of constant vertical column density, and the colored curves show isodensity contours for the CO lower level, with the upper heavy dashed curve showing the layer where the line becomes optically thick. (The lower right region shows where $\mathrm{CO}$ freezes out. This model produces the emission line profile shown in the right panel; the sharpness of the peaks and overall profile shape (modeled spatially) provides an indication that any turbulent broadening must be small. From Flaherty et al. (2015).

Clure et al. 2015), but these observations undoubtedly trace outer regions at tens of $\mathrm{AU}$ or more.

\subsection{Observational limits on turbulence}

The magnitude of turbulence in planet-forming disks plays an important role in allowing (or preventing) coagulation/growth of solids, as well as affecting both mass and angular momentum transport. Unfortunately observational constraints on turbulence are extremely limited. Observations of the firstovertone lines of $\mathrm{CO}$ have yielded estimates of transsonic turbulence in the upper layers of disks at radii $\lesssim 1 \mathrm{AU}$ in a few systems, but these objects are either relatively luminous (Najita et al. 1996, 2009) or very rapidly accreting (Hartmann et al. 2004), such that temperatures are likely high enough to support the magnetorotational instability (MRI) via thermal ionization (\$3). The most detailed limits so far for colder disk regions come from the recent ALMA study of the large disk surrounding the $L \sim 35 L_{\odot}$ A-type star HD 163296 (Flaherty et al. 2015). Using a sequence of CO isotopologues to deal with issues of temperature structure, excitation, and optical depth, Flaherty et al. conclude that non-thermal motions in the upper disk layers are no more than about $3-6 \%$ of the local sound speed at $R \gtrsim 30$ AU (see Figure 7), an order of magnitude less than expected for full MRI turbulence, though still significant enough to play a role in mixing and transport of disk materials.

\section{Turbulence, winds, and mass and angular momentum transport}

Turbulence can play an important role in disk density and temperature structures via its effects on viscous dissipation and mass transport. As discussed in 
2.2 to maintain observed accretion rates over evolutionary timescales seems to imply transport of a significant fraction of initial disk masses into the central star. Turbulence can also affect the amount of heating by light from the central star if it stirrs up small dust grains (Dullemond and Dominik 2005), enhancing their vertical scale height relative to the gas (Fromang and Papaloizou 2006).

In the case of fully-ionized disks, the magneto-rotational instability (MRI: Balbus and Hawley 1998) produces efficient turbulent angular momentum transport, with viscosities parameterized by the parameter $\alpha \sim 10^{-1}-10^{-2}$ (\$1.4). However, as they are comparatively dense and cold, protoplanetary disks (PPDs) are generally expected to have extremely low levels of ionisation deep in their interiors (see Turner et al. 2014, for a recent review). Only inner $(\lesssim 0.3 \mathrm{AU})$ regions of protoplanetary disks with higher temperatures and surface layers ionised by hard radiation (such as FUV photons, X-rays, cosmic rays) from the magnetically active central star (see section 2.5 in Turner et al. 2014), or potentially from an actively star-forming nearby cluster environment (Kalyaan et al. 2015), can result in effective coupling of the ions with the neutral gas. Cosmic rays could be especially important in ionising gas, particularly in the outer disk where column densities are low, but deflection by the stellar wind or magnetic fields might prevent them from becoming important (Cleeves et al. 2013).

The ionising events from external sources are balanced by recombination with gas-phase ions and, in the presence of sub-micron-sized dust, by absorption on the grain surface. Based on estimates of the abundance of free electrons and the collision regimes characteristic of various regions within the disc, it is now commonly accepted that all three non-ideal MHD effects, namely, Ohmic resistivity, ambipolar diffusion (AD), and the Hall term have to be considered in the generalised Ohm's law to arrive at sound conclusions for the overall dynamical state of PPDs (e.g. Salmeron and Wardle 2003, Bai 2011).

\subsection{Ambipolar diffusion affected laminar disks}

The inclusion of ambipolar diffusion has strongly modified the original layered model assuming only Ohmic resistivity, with an MRI-active upper disk layers providing the angular momentum transport and viscous heating (Gammie 1996). assumed only Ohmic resistivity, with Studies by Bai and Stone (2013), Bai (2013), and Simon et al. (2013a b) show that in the absence of net vertical flux, the MRI is typically suppressed in wide radial stretches of typical T Tauri disks and standard solar-nebular models, and the resulting state is a laminar flow with negligible angular momentum transport. Moreover, otherwise laminar regions in the inner disk (roughly speaking $R \simeq 1-20 \mathrm{au}$ ) can launch a magnetocentrifugal wind within the FUV-ionised surface layers. Such ADassisted magnetic winds were actually explored in prescient work by Königl (1989) and Wardle and Königl (1993), who developed self-simular steady disk solutions matching to the wind solutions of Blandford and Payne (1982), and illustrate many of the main features of subsequent numerical simulations Bai 
and Stone (2013); Simon et al. (2013a); Lesur et al. (2014). However, due to technical limitations (such as finite computational volumes), mass loading and mass loss rates are uncertain. Since the role of the far-UV ionised upper disk surface is crucial (Perez-Becker and Chiang 2011), the shielding of the required energetic photons needs to be assessed to corroborate the foundation of the mechanism.

A noteworthy feature of AD laminar accretion disks is the existence of thin current layers, which have both been found in the local models of Bai and Stone (2013), and the global ones of Gressel et al. (2015). These current layers can produce strong radial streams of material (at $\sim 30-40 \%$ of the sound speed), and can potentially provide non-negligible amounts of radial mass transport. As has recently been argued by Bai et al. (2016), laminar MHD winds can in principle team up with photoevaporative acceleration (e.g. Alexander et al. 2006) to remove mass and limit disk lifetimes (see 3.3 . .

\subsection{Magnetic self-organisation and the Hall effect}

Although suggestions of the importance of the Hall effect in PPDs have been made for some time (e.g., Wardle and Königl 1993), it has only recently been included in detailed numerical simulations. Complicating matters, the dynamical outcome of the Hall effect depends upon the mutual sign of the rotation vector (represented by $\boldsymbol{\Omega}$ ) and large-scale magnetic flux (represented by $\mathbf{B}$ ). The Hall drift has the effect to either boost (in the case of $\boldsymbol{\Omega} \cdot \mathbf{B}>0$ ) or impede (conversely for $\boldsymbol{\Omega} \cdot \mathbf{B}<0$ ) linear growth (Lesur et al. 2014, Bai 2015) of the MRI. Notably, with a somewhat modified ionisation model, Simon et al. (2015) found bursty behaviour in the latter case.

While the MRI relies on the combination of field induction and plasma inertia (described by perturbations $\delta \mathbf{v}$ and $\delta \mathbf{B}$ ), there is an even simpler instability that strictly only relies on the induction equation (and perturbations $\delta \mathbf{B}$ ) this has been termed the Hall-shear instability (HSI, Kunz 2008). As studied for the case of a simplified Hall coefficient that results in a fixed length scale, $l_{\mathrm{H}} \equiv \eta_{\mathrm{H}} / v_{\mathrm{A}}$, the HSI can lead to self-organisation behaviour (for $l_{\mathrm{H}} \simeq H$ ) and, depending on the strength of the effect, creates azimuthally confined zonal bands and/or vortices.

MRI in the context of Hall-MHD has so far been studied in unstratified (Sano and Stone 2002, Kunz and Lesur 2013) and stratified (Lesur et al. 2014, Bai 2014) shearing-box models, and in unstratified cylindrical disk models (O'Keeffe and Downes 2014; Béthune et al. 2016). The microphysics of the Hall effect is complicated by many uncertainties, such as the population of small grains and their charge.

The presence of strong self-organising azimuthal field belts naturally begs the question of buoyant stability of these configurations, the study of which will demand vertically-stratified global disk models. As emphasised by $\mathrm{Xu}$ and Bai (2016), and demonstrated by Gressel et al. (2015) in the context of $\mathrm{AD}$, the field-strength dependence of diffusion coefficients, that is $\eta_{\mathrm{H}} \sim$ 
$B$ and $\eta_{\mathrm{A}} \sim B^{2}$, opens the possibility of highly non-trivial evolution of the system once it reaches non-linear field amplitudes. Finally, a spatial gradient in the coefficients (for instance at the FUV interface as shown by Gressel et al. 2015) can produce non-negligible electromotive forces (potentially leading to significant dynamical effects) all by itself.

\subsection{Photoevaporative mass-loss}

In addition to magnetically driven winds and jets, protoplanetary disks can also lose mass through photoevaporation. Stellar UV and/or X-ray irradiation heats the disk surface to $\sim 10^{3}-10^{4} \mathrm{~K}$, and beyond some critical radius (typically 1-10AU) the hot gas is unbound and escapes as a wind (e.g. Alexander et al. 2014; Gorti et al. 2016). Photoevaporative disk winds have now been observed directly in a number of objects (e.g., Pascucci and Sterzik 2009, Sacco et al. 2012), and are probably responsible for clearing the outer disk. If a magnetic field is present, the thermal pressure can complement the magnetic acceleration to enhance mass loss and remove angular momentum (\$3.1).

The impact of photoevaporative mass-loss on volatiles, and water in particular, is less clear. The wind is typically launched from the upper layers of the disk, and can entrain particles at least as large as $\mu m$-size grains (Takeuchi et al. 2005). Moreover, the mass-loss is concentrated at a radius ( $\sim 1-10 \mathrm{AU})$ comparable to the snow line, and could therefore carry away water in both gaseous and solid phases. The impact on the disk's chemical composition therefore depends critically on its radial and (especially) vertical structure, but there has been relatively little exploration of this issue in the literature. Most relevant is the work of Guillot and Hueso (2006), who showed that photoevaporation can play a role in enriching the solar system gas giants in noble gases. However, in light of our improved understanding of both mass-loss and turbulence, more detailed modelling is required if we are to assess the importance of this process more critically.

\subsection{Implications for disk structure, turbulence and heating}

Figure 8 summarizes the recent ideas about PPD structure, turbulence, and transport. In the inner disk, thermal ionization results in MRI transport. Beyond about $0.3 \mathrm{AU}$ (for a low-mass central star), ionization levels are high only in surface layers due to the limited penetration of X-rays (and maybe cosmic rays). Magnetic fields threading the disk can produce outflows (probably enhanced by photoevaporative heating), resulting in angular momentum transport and accretion in thin layers where AD begins to become important. The Hall effect can produce strong azimuthal fields, and may result in qualitatively different behavior depending upon the orientation of the magnetic field relative to the angular momentum vector.

It must be emphasized that the operation of all these non-ideal MHD processes depend upon the amount to which the disk is able to keep magnetic 


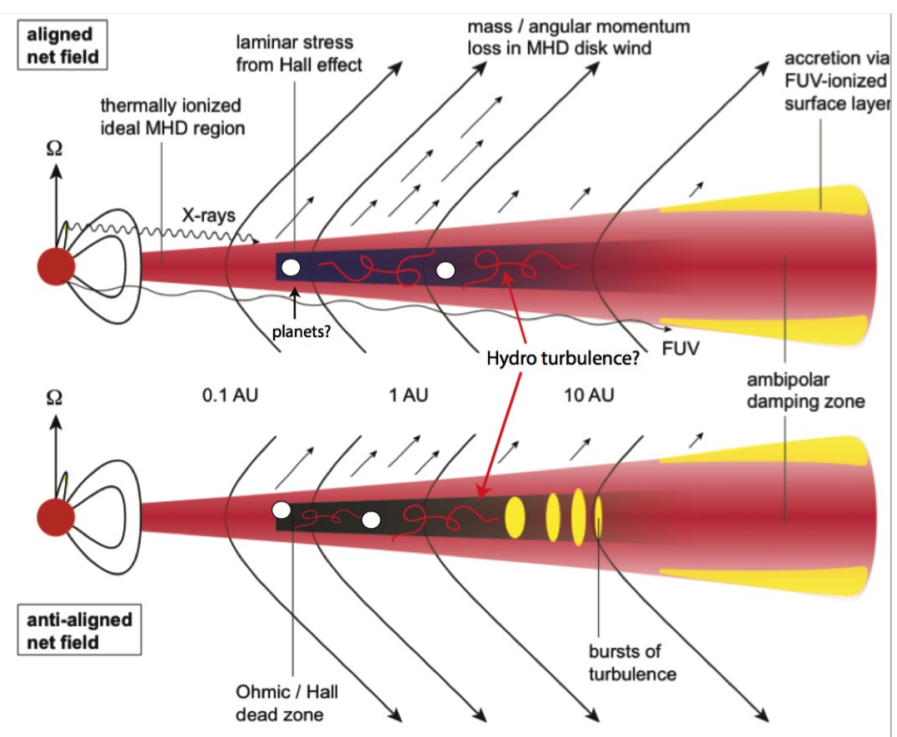

Fig. 8 Schematic disk structure suggested for protoplanetary disks with non-ideal MHD transport, including the Hall effect. Modified from Simon et al. (2015)

flux, which is poorly constrained (Okuzumi et al. 2014, Guilet and Ogilvie 2014), and will likely depend on the level of disk turbulence, as well as the effective magnetic Prandtl number (Fromang and Stone 2009) of the turbulent flow. This has helped stimulate interest in hydrodynamic instabilities which can operate in the absence of magnetic stresses, such as the vertical shear instability (Nelson et al. 2013; Stoll and Kley 2016), the convective overstability instability (Lyra |2014 |Klahr and Hubbard |2014), the "zombie vortex" instability (Marcus et al.(2015); but see Lesur and Latter (2016)). These instabilities (which depend critically on rates of radiative relaxation; Malygin et al. (2017)) generally result in low viscosities and/or transport, with $\alpha \sim 10^{-4}$, but this could be enough to produce important effects on mass transport and dust coagulation. Finally, it has even been suggested that, in very low viscosity disks, planets (super-Earths) can actually produce strong enough spiral waves which reduce their inward migration while producing observable accretion rates (Fung and Chiang 2017). Obviously our understanding of PPDs is highly uncertain; tentatively, one may conclude that both theory and observations are driving us to a picture in which most of the disks are subject to low levels of turbulence.

\section{A Framework for Water Evolution in Protoplanetary Disks}

As discussed above, there are still significant uncertainties in the extent to which the different processes described above impact how protoplanetary disks 
are structured and evolve. A greater complication arises as we work to understand how all of these processes operate simultaneously to shape the distribution of water in the disk. That is, as solids grow to different sizes, with water being incorporated into bodies with differing dynamical properties, the distribution of water ice and vapor will continuously evolve over the lifetime of the protoplanetary disk. Here we outline a framework for understanding how this evolution may proceed, and the impact it may have on different aspects of planet formation.

Early on in disk history, dust grains will begin to coagulate and form larger and larger solids. Outside the snow line $\left(r>R_{\mathrm{SL}}\right)$, water ice-bearing solids will concentrate at the disk midplane as a result of settling, resulting in enhanced $\mathrm{H}_{2} \mathrm{O} / \mathrm{H}_{2}$ values. Water vapor at high altitudes can diffuse downward and freezeout on these solids, only returning again as the fine-dust that remains strongly coupled to the gas is lofted to high altitudes allowing desorption to occur again. As the abundance of fine grained dust becomes depleted, the amount of vapor present at high altitudes in the disk diminishes, effectively lowering the height of the snow line. This is a result of the water vapor concentration decreasing, meaning the desorption flux will exceed the adsorption flux through more of the disk (Krijt and Ciesla 2016).

As radial drift becomes important, with solids millimeters and centimeters in size marching inwards, the location of the snow line will become blurred. The most rapidly drifting solids, those millimeters to meters in scale, have drift timescales that are short compared to their sublimation timescale (Cyr et al. 1998; (Piso et al. 2015). As a result, icy solids may be found inside of $R_{\mathrm{SL}}$. The exact distance to which icy solids would survive drifting inside the snow line depends on the details of the temperature gradient in the disk, but may be as much as 1-2 AU.

As these icy solids sublimate, the water will return to the gas phase. Because the inward drift of solids can be rapid (inward drift rates will be of order $\mathrm{cm} / \mathrm{s}$ to $\mathrm{m} / \mathrm{s}$ relative to the gas), the delivery of water vapor to the inner disk can be much faster than the rate at which it is redistributed by radial diffusion. This imbalance will lead to water vapor becoming enhanced in the inner disk, with concentrations increasing either until the outward flux of water vapor via diffusion equals the inward flux from drifting pebbles, or until inward drift ceases either because the pebbles are accreted by growing planets or are no longer able to form due to the depleted reservoir of solids in the outer disk (Cuzzi and Zahnle 2004, Ciesla and Cuzzi 2006, Estrada et al. 2016). Detailed models suggest that water vapor enhancements of a 3-5× over the "canonical" value could be seen inside the snow line (Ciesla and Cuzzi 2006, Estrada et al. 2016) 1

Once the inward drift of icy solids slows, vapor diffusion will remove water vapor by carrying it beyond the snow line to freeze-out again. If the ice is locked

1 Ciesla and Cuzzi (2006) used an incorrect form of the equation describing the diffusive redistribution of materials; correction of this term leads to changes in the predicted concentrations of a factor of a few. Estrada et al. (2016) provided a more rigorous treatment of growth and transport and found that the qualitative relationships and behaviors hold true. 
up efficiently beyond the snow line so that it cannot drift inward again, then the inner disk will become continuously depleted in water vapor throughout the rest of its lifetime (Stevenson and Lunine 1988; Cyr et al. 1998, Cuzzi and Zahnle 2004: Ciesla and Cuzzi 2006). This diffusion serves to increase the ice concentration just beyond the snow line, meaning that the the region just beyond the snow line is expected to become enhanced in solids. The migration of the snow line over time, as a result of the evolution of the disk, would set exactly where such enhancements would occur. The particulars of disk evolution may change things, as if the net flows of gas around the snow line, depending on their orientation, could carry the water vapor enhanced/depleted gas inside the snow line outward/inward, or the ice enhanced/depleted gas inward, which would impact the overall distribution of water in the disk over time.

\subsection{Implications of Water Evolution for Planet Formation}

The varying dynamic properties of water-bearing species will lead to continuous evolution in the distribution of water within the disk, both in the gas and solid phases. The magnitude and timescale for this evolution will depend on many uncertain parameters, including the efficiency of particle growth and the level of turbulence (diffusion) within the disk. There is, however, a number of features in full-born and proto-planetary systems that may be linked to this evolution which we discuss here. By understanding these relationships, perhaps we can begin to evaluate the uncertainties in the parameters which describe protoplanetary disk processes.

The concentration of solids that would develop just outside of the snow line due to the inward drift of ices from greater heliocentric distances and the outward diffusion of ices may serve to seed the early stages of giant planet formation. Stevenson and Lunine (1988) originally suggested that the outward diffusion of vapor from the inner Solar System to beyond the assumed snow line location of $5 \mathrm{AU}$ could have been a catalyst for the formation of Jupiter, as a massive core could grow more readily from the higher concentration of mass then expected. While disk temperatures are predicted to be such for the snow line around a solar mass star to have been located closer to 1-3 AU, it is also possible that Jupiter did not form at its current orbit, and may have migrated outwards to its current location at some later point (e.g. Walsh et al. 2011). Further, a general view for many large exoplanets close to their host stars is that they formed further out in their protoplanetary disk then migrated inwards to where we observe them today; perhaps this was due to a very distant snow line early in disk evolution when mass accretion rates (viscous dissipation) were high (assuming there is significant viscosity; 83 .)

The fluctuating water conditions in the inner protoplanetary disk may play a role in shaping the chemistry and mineralogy of planetary building blocks that form there. Cuzzi and Zahnle (2004) originally suggested that water vapor levels could be enhanced by many orders of magnitude by the inward drift 
of solids, which may have explained how iron-bearing silicates were able to form (in the absence of extreme oxygen enhancements, Fe is expected only to exist in metallic form (e.g. Fedkin and Grossman 2006)). However, it appears that such enhancements are limited to just a factor of a few, due to finite reservoirs of ices and the difficulty in growing large populations of very rapidly drifting solids (Ciesla and Cuzzi 2006; Estrada et al. 2016). Later water vapor depletions would aid in the development of very reducing conditions, and may be required to explain the mineralogy observed in enstatite chondrites $(\mathrm{Cyr}$ et al. 1999, Hutson and Ruzicka 2000).

It is worth noting that the evolution described here would not be limited to water alone. $\mathrm{CO}$ will freeze-out onto solids in protoplanetary disks at temperatures below $\sim 20 \mathrm{~K}$ (Qi et al. 2013), and will be subjected to the same settle/drift-adsorb/desorb cycling described above. This will also lead to fluctuating distributions of $\mathrm{CO}$ in protoplanetary disks, something that must be remembered as this molecule is readily observed and used to trace gas evolution and mass (Williams and Best 2014).

\section{Final Remarks}

Theoretical expectations of the positions of water snow lines in protoplanetary disks are unclear, due to the large uncertainties in dust opacities and the amount of viscous dissipation present. Unfortunately, observations so far do not provide meaningful constraints because of limited angular resolution and the significant dust optical depths in inner disks. Further progress might result from longer-wavelength interferometry to detect opacity transitions due to ice that arise from the fact that water ice on the surface of grains makes them more sticky, and thus able to grow to larger sizes than the rocky grains inside of the snow line (Banzatti et al. 2015). Additionally, more sophisticated disk models with advanced chemistry and radiative transfer is needed to take advantage of the current improvements in spatially-resolved dust and molecular emission in disks to provide further insight into the distribution of water. For now, all that can be said with reasonable confidence is that snow lines in protoplanetary disks around solar-type stars should lie in the range of a few to one $\mathrm{AU}$, and move in with time as accretion slows and dust opacities decrease.

The existence of transitional disks, with large holes cleared of small dust at early ages, suggests that some planetesimal, embryo, or planet formation stages might occur during optically-thin phases of inner disks. If the formation of Jupiter could have produced such a transitional disk structure by preventing small dust from entering the inner Solar System, the effectively optically-thin temperature estimate of equation (2) might be relevant after all.

Acknowledgements The research of LH was supported by the University of Michigan and in part by NASA grant NNX16AB46G. FC acknowledges support from NASA's Exobiology and Outer Planets Research Programs (NNX12AD59G and NNX14AQ17G). OG has received funding from the European Research Council (ERC) under the European Union's Horizon 2020 research and innovation programme (grant agreement No 638596). RA has 
received funding from the European Research Council (ERC) under the European Union's Horizon 2020 research and innovation programme (grant agreement No 681601), and also acknowledges support from the Leverhulme Trust through a Philip Leverhulme Prize.

\section{References}

Y. Abe, E. Ohtani, T. Okuchi, K. Righter, M. Drake, Water in the Early Earth, ed. by R.M. Canup, K. Righter, et al.2000, pp. 413-433

R.D. Alexander, C.J. Clarke, J.E. Pringle, Photoevaporation of protoplanetary discs - I. Hydrodynamic models. MNRAS 369, 216-228 (2006)

R. Alexander, I. Pascucci, S. Andrews, P. Armitage, L. Cieza, The Dispersal of Protoplanetary Disks. Protostars and Planets VI, 475-496 (2014)

S.M. Andrews, J.P. Williams, Circumstellar Dust Disks in Taurus-Auriga: The Submillimeter Perspective. Ap. J. 631, 1134-1160 (2005)

S.M. Andrews, D.J. Wilner, A.M. Hughes, C. Qi, K.A. Rosenfeld, K.I. Öberg, T. Birnstiel, C. Espaillat, L.A. Cieza, J.P. Williams, S.-Y. Lin, P.T.P. Ho, The TW Hya Disk at 870 $\mu \mathrm{m}$ : Comparison of CO and Dust Radial Structures. Ap. J. 744, 162 (2012)

X.-N. Bai, Magnetorotational-instability-driven Accretion in Protoplanetary Disks. Ap. J. 739, 50 (2011)

X.-N. Bai, Wind-driven Accretion in Protoplanetary Disks. II. Radial Dependence and Global Picture. Ap. J. 772, 96 (2013)

X.-N. Bai, Hall-effect-Controlled Gas Dynamics in Protoplanetary Disks. I. Wind Solutions at the Inner Disk. Ap. J. 791, 137 (2014)

X.-N. Bai, Hall Effect Controlled Gas Dynamics in Protoplanetary Disks. II. Full 3D Simulations toward the Outer Disk. Ap. J. 798, 84 (2015)

X.-N. Bai, J.M. Stone, Wind-driven Accretion in Protoplanetary Disks. I. Suppression of the Magnetorotational Instability and Launching of the Magnetocentrifugal Wind. Ap. J. 769, 76 (2013)

X.-N. Bai, J. Ye, J. Goodman, F. Yuan, Magneto-thermal Disk Winds from Protoplanetary Disks. Ap. J. 818, $152(2016)$

S.A. Balbus, J.F. Hawley, Instability, turbulence, and enhanced transport in accretion disks. Reviews of Modern Physics 70, 1-53 (1998)

A. Banzatti, P. Pinilla, L. Ricci, K.M. Pontoppidan, T. Birnstiel, F. Ciesla, Direct Imaging of the Water Snow Line at the Time of Planet Formation using Two ALMA Continuum Bands. Ap. J. Lett. 815, 15 (2015)

E.A. Bergin, L.I. Cleeves, U. Gorti, K. Zhang, G.A. Blake, J.D. Green, S.M. Andrews, N.J. Evans II, T. Henning, K. Öberg, K. Pontoppidan, C. Qi, C. Salyk, E.F. van Dishoeck, An old disk still capable of forming a planetary system. Nature 493, 644-646 (2013)

E. Bergin, N. Calvet, P. D'Alessio, G.J. Herczeg, The Effects of UV Continuum and Ly $\alpha$ Radiation on the Chemical Equilibrium of T Tauri Disks. Ap. J. Lett. 591, 159-162 (2003)

W. Béthune, G. Lesur, J. Ferreira, Self-organisation in protoplanetary discs. Global, nonstratified Hall-MHD simulations. Astron. Astrophys. 589, 87 (2016)

T. Birnstiel, C.W. Ormel, C.P. Dullemond, Dust size distributions in coagulation/fragmentation equilibrium: numerical solutions and analytical fits. Astron. Astrophys. 525, 11 (2011)

B. Bitsch, A. Johansen, M. Lambrechts, A. Morbidelli, The structure of protoplanetary discs around evolving young stars. Astron. Astrophys. 575, 28 (2015)

R.D. Blandford, D.G. Payne, Hydromagnetic flows from accretion discs and the production of radio jets. MNRAS 199, 883-903 (1982)

S.M. Blevins, K.M. Pontoppidan, A. Banzatti, K. Zhang, J.R. Najita, J.S. Carr, C. Salyk, G.A. Blake, Measurements of water surface snow lines in classical protoplanetary disks. ArXiv e-prints (2015)

S.D. Brittain, J.R. Najita, J.S. Carr, Near infrared high resolution spectroscopy and spectroastrometry of gas in disks around Herbig Ae/Be stars. Astrophysics and Space Science 357, 54 (2015) 
N. Calvet, P. D'Alessio, L. Hartmann, D. Wilner, A. Walsh, M. Sitko, Evidence for a Developing Gap in a 10 Myr Old Protoplanetary Disk. Ap. J. 568, 1008-1016 (2002)

J.S. Carr, J.R. Najita, Organic Molecules and Water in the Inner Disks of T Tauri Stars. Ap. J. 733, 102 (2011)

E.I. Chiang, P. Goldreich, Spectral Energy Distributions of T Tauri Stars with Passive Circumstellar Disks. Ap. J. 490, 368-376 (1997)

F.J. Ciesla, J.N. Cuzzi, The evolution of the water distribution in a viscous protoplanetary disk. Icarus 181, 178-204 (2006)

L.A. Cieza, S. Casassus, J. Tobin, S.P. Bos, J.P. Williams, S. Perez, Z. Zhu, C. Caceres, H. Canovas, M.M. Dunham, A. Hales, J.L. Prieto, D.A. Principe, M.R. Schreiber, D. Ruiz-Rodriguez, A. Zurlo, Imaging the water snow-line during a protostellar outburst. Nature 535, 258-261 (2016). doi:10.1038/nature18612

L.I. Cleeves, F.C. Adams, E.A. Bergin, Exclusion of Cosmic Rays in Protoplanetary Disks: Stellar and Magnetic Effects. Ap. J. 772, 5 (2013)

L.I. Cleeves, E.A. Bergin, C.M.O.. Alexander, F. Du, D. Graninger, K.I. Öberg, T.J. Harries, The ancient heritage of water ice in the solar system. Science 345, 1590-1593 (2014)

J.N. Connelly, M. Bizzarro, A.N. Krot, Å. Nordlund, D. Wielandt, M.A. Ivanova, The Absolute Chronology and Thermal Processing of Solids in the Solar Protoplanetary Disk. Science 338, 651 (2012)

J.N. Cuzzi, K.J. Zahnle, Material Enhancement in Protoplanetary Nebulae by Particle Drift through Evaporation Fronts. Ap. J. 614, 490-496 (2004)

J.N. Cuzzi, A.R. Dobrovolskis, J.M. Champney, Particle-gas dynamics in the midplane of a protoplanetary nebula. Icarus 106, 102 (1993). doi:10.1006/icar.1993.1161

K.E. Cyr, W.D. Sears, J.I. Lunine, Distribution and Evolution of Water Ice in the Solar Nebula: Implications for Solar System Body Formation. Icarus 135, 537-548 (1998)

K.E. Cyr, C.M. Sharp, J.I. Lunine, Effects of the redistribution of water in the solar nebula on nebular chemistry. J. Geophys. Res. 104, 19003-19014 (1999)

P. D'Alessio, N. Calvet, L. Hartmann, Accretion Disks around Young Objects. III. Grain Growth. Ap. J. 553, 321-334 (2001)

P. D'Alessio, J. Cantö, N. Calvet, S. Lizano, Accretion Disks around Young Objects. I. The Detailed Vertical Structure. Ap. J. 500, 411-427 (1998)

S.J. Desch, Mass Distribution and Planet Formation in the Solar Nebula. Ap. J. 671, 878$893(2007)$

C.P. Dullemond, C. Dominik, Dust coagulation in protoplanetary disks: A rapid depletion of small grains. Astron. Astrophys. 434, 971-986 (2005)

C. Espaillat, N. Calvet, P. D’Alessio, J. Hernández, C. Qi, L. Hartmann, E. Furlan, D.M. Watson, On the Diversity of the Taurus Transitional Disks: UX Tauri A and LkCa 15. Ap. J. Lett. 670, 135-138 (2007)

C. Espaillat, J. Muzerolle, J. Najita, S. Andrews, Z. Zhu, N. Calvet, S. Kraus, J. Hashimoto, A. Kraus, P. D'Alessio, An Observational Perspective of Transitional Disks. Protostars and Planets VI, 497-520 (2014)

P.R. Estrada, J.N. Cuzzi, D.A. Morgan, Global Modeling of Nebulae with Particle Growth, Drift, and Evaporation Fronts. I. Methodology and Typical Results. Ap. J. 818, 200 (2016)

D. Fedele, M.E. van den Ancker, T. Henning, R. Jayawardhana, J.M. Oliveira, Timescale of mass accretion in pre-main-sequence stars. Astron. Astrophys. 510, 72 (2010)

A.V. Fedkin, L. Grossman, The Fayalite Content of Chondritic Olivine: Obstacle to Understanding the Condensation of Rocky Material, ed. by D.S. Lauretta, H.Y. McSween 2006, pp. 279-294

K.M. Flaherty, A.M. Hughes, K.A. Rosenfeld, S.M. Andrews, E. Chiang, J.B. Simon, S. Kerzner, D.J. Wilner, Weak Turbulence in the HD 163296 Protoplanetary Disk Revealed by ALMA CO Observations. Ap. J. 813, 99 (2015)

S. Fromang, J. Papaloizou, Dust settling in local simulations of turbulent protoplanetary disks. Astron. Astrophys. 452, 751-762 (2006)

S. Fromang, J.M. Stone, Turbulent resistivity driven by the magnetorotational instability. Astron. Astrophys. 507, 19-28 (2009)

J. Fung, E. Chiang, Save the Planet, Feed the Star: How Super-Earths Survive Migration and Drive Disk Accretion. Ap. J. 839, 100 (2017). doi:10.3847/1538-4357/aa6934 
E. Furlan, L. Hartmann, N. Calvet, P. D’Alessio, R. Franco-Hernández, W.J. Forrest, D.M. Watson, K.I. Uchida, B. Sargent, J.D. Green, L.D. Keller, T.L. Herter, A Survey and Analysis of Spitzer Infrared Spectrograph Spectra of T Tauri Stars in Taurus. Ap. J. Suppl. Ser. 165, 568-605 (2006)

C.F. Gammie, Layered Accretion in T Tauri Disks. Ap. J. 457, 355 (1996)

P. Garaud, D.N.C. Lin, The Effect of Internal Dissipation and Surface Irradiation on the Structure of Disks and the Location of the Snow Line around Sun-like Stars. Ap. J. 654, 606-624 (2007)

A.E. Glassgold, R. Meijerink, J.R. Najita, Formation of Water in the Warm Atmospheres of Protoplanetary Disks. Ap. J. 701, 142-153 (2009)

U. Gorti, R. Liseau, Z. Sándor, C. Clarke, Disk Dispersal: Theoretical Understanding and Observational Constraints. Space Sci.Rev(2016)

O. Gressel, N.J. Turner, R.P. Nelson, C.P. McNally, Global Simulations of Protoplanetary Disks With Ohmic Resistivity and Ambipolar Diffusion. Ap. J. 801, 84 (2015)

J. Guilet, G.I. Ogilvie, Global evolution of the magnetic field in a thin disc and its consequences for protoplanetary systems. MNRAS 441, 852-868 (2014)

T. Guillot, R. Hueso, The composition of Jupiter: sign of a (relatively) late formation in a chemically evolved protosolar disc. MNRAS 367, 47-51 (2006)

L. Hartmann, S.J. Kenyon, The FU Orionis Phenomenon. Annu. Rev. Astron. Astrophys. 34, 207-240 (1996)

L. Hartmann, G. Herczeg, N. Calvet, Accretion onto Pre-Main-Sequence Stars. Annu. Rev. Astron. Astrophys. 54, 135-180 (2016). doi:10.1146/annurev-astro-081915-023347

L. Hartmann, K. Hinkle, N. Calvet, High-Resolution Near-Infrared Spectroscopy of FU Orionis Objects. Ap. J. 609, 906-916 (2004)

C. Hayashi, Structure of the Solar Nebula, Growth and Decay of Magnetic Fields and Effects of Magnetic and Turbulent Viscosities on the Nebula. Progress of Theoretical Physics Supplement 70, 35-53 (1981)

J. Hernández, L. Hartmann, N. Calvet, R.D. Jeffries, R. Gutermuth, J. Muzerolle, J. Stauffer, A Spitzer View of Protoplanetary Disks in the $\gamma$ Velorum Cluster. Ap. J. 686, 1195-1208 (2008)

I. Hubeny, Vertical structure of accretion disks - A simplified analytical model. Ap. J. 351, 632-641 (1990)

M. Hutson, A. Ruzicka, A multi-step model for the origin of E3 (enstatite) chondrites. Meteoritics and Planetary Science 35, 601-608 (2000)

L. Ingleby, N. Calvet, E. Bergin, A. Yerasi, C. Espaillat, G. Herczeg, E. Roueff, H. Abgrall, J. Hernández, C. Briceño, I. Pascucci, J. Miller, J. Fogel, L. Hartmann, M. Meyer, J. Carpenter, N. Crockett, M. McClure, Far-Ultraviolet $\mathrm{H}_{2}$ Emission from Circumstellar Disks. Ap. J. Lett. 703, 137-141 (2009)

L. Ingleby, N. Calvet, J. Hernández, L. Hartmann, C. Briceno, J. Miller, C. Espaillat, M. McClure, The Evolution of Accretion in Young Stellar Objects: Strong Accretors at 3-10 Myr. Ap. J. 790, 47 (2014)

A. Kalyaan, S.J. Desch, N. Monga, External Photoevaporation of the Solar Nebula. II. Effects on Disk Structure and Evolution with Non-uniform Turbulent Viscosity due to the Magnetorotational Instability. Ap. J. 815, 112 (2015)

G.M. Kennedy, S.J. Kenyon, Planet Formation around Stars of Various Masses: The Snow Line and the Frequency of Giant Planets. Ap. J. 673, 502-512 (2008)

S.J. Kenyon, L. Hartmann, Spectral energy distributions of T Tauri stars - Disk flaring and limits on accretion. Ap. J. 323, 714-733 (1987)

H. Klahr, A. Hubbard, Convective Overstability in Radially Stratified Accretion Disks under Thermal Relaxation. Ap. J. 788, 21 (2014)

A. Königl, Self-similar models of magnetized accretion disks. Ap. J. 342, 208-223 (1989)

S. Krijt, F.J. Ciesla, Tracing Solid Growth and Vapor Distributions in Vertical Columns of Protoplanetary Disks. Ap. J.Submitted (2016)

T.S. Kruijer, M. Touboul, M. Fischer-Gödde, K.R. Bermingham, R.J. Walker, T. Kleine, Protracted core formation and rapid accretion of protoplanets. Science 344, 1150-1154 (2014)

M.W. Kunz, On the linear stability of weakly ionized, magnetized planar shear flows. $M N$ RAS 385, 1494-1510 (2008) 
M.W. Kunz, G. Lesur, Magnetic self-organization in Hall-dominated magnetorotational turbulence. MNRAS 434, 2295-2312 (2013)

G. Lesur, M.W. Kunz, S. Fromang, Thanatology in protoplanetary discs. The combined influence of Ohmic, Hall, and ambipolar diffusion on dead zones. Astron. Astrophys. 566, 56 (2014)

G.R.J. Lesur, H. Latter, On the survival of zombie vortices in protoplanetary discs. $M N R A S$ 462, 4549-4554 (2016). doi:10.1093/mnras/stw2172

K. Lodders, Solar System Abundances and Condensation Temperatures of the Elements. Ap. J. 591, 1220-1247 (2003)

W. Lyra, Convective Overstability in Accretion Disks: Three-dimensional Linear Analysis and Nonlinear Saturation. Ap. J. 789, 77 (2014)

M.G. Malygin, H. Klahr, D. Semenov, T. Henning, C.P. Dullemond, Efficiency of thermal relaxation by radiative processes in protoplanetary discs: constraints on hydrodynamic turbulence. ArXiv e-prints (2017)

P.S. Marcus, S. Pei, C.-H. Jiang, J.A. Barranco, P. Hassanzadeh, D. Lecoanet, Zombie Vortex Instability. I. A Purely Hydrodynamic Instability to Resurrect the Dead Zones of Protoplanetary Disks. Ap. J. 808, 87 (2015). doi:10.1088/0004-637X/808/1/87

M.K. McClure, C. Espaillat, N. Calvet, E. Bergin, P. D’Alessio, D.M. Watson, P. Manoj, B. Sargent, L.I. Cleeves, Detections of Trans-Neptunian Ice in Protoplanetary Disks. Ap. J. 799, $162(2015)$

J.R. Najita, S.E. Strom, J. Muzerolle, Demographics of transition objects. MNRAS 378, 369-378 (2007)

J.R. Najita, G.W. Doppmann, J.S. Carr, J.R. Graham, J.A. Eisner, High-Resolution K-Band Spectroscopy of MWC 480 and V1331 Cyg. Ap. J. 691, 738-748 (2009)

J. Najita, J.S. Carr, A.E. Glassgold, F.H. Shu, A.T. Tokunaga, Kinematic Diagnostics of Disks around Young Stars: CO Overtone Emission from WL 16 and 1548C27. Ap. J. 462, 919 (1996)

R.P. Nelson, O. Gressel, O.M. Umurhan, Linear and non-linear evolution of the vertical shear instability in accretion discs. MNRAS 435, 2610-2632 (2013)

W. O'Keeffe, T.P. Downes, Multifluid simulations of the magnetorotational instability in protostellar discs. MNRAS 441, 571-581 (2014)

S. Okuzumi, T. Takeuchi, T. Muto, Radial Transport of Large-scale Magnetic Fields in Accretion Disks. I. Steady Solutions and an Upper Limit on the Vertical Field Strength. Ap. J. 785, 127 (2014)

J.E. Owen, B. Ercolano, C.J. Clarke, Protoplanetary disc evolution and dispersal: the implications of X-ray photoevaporation. MNRAS 412, 13-25 (2011)

I. Pascucci, M. Sterzik, Evidence for Disk Photoevaporation Driven by the Central Star. Ap. J. 702, 724-732 (2009)

L.M. Pérez, J.M. Carpenter, C.J. Chandler, A. Isella, S.M. Andrews, L. Ricci, N. Calvet, S.A. Corder, A.T. Deller, C.P. Dullemond, J.S. Greaves, R.J. Harris, T. Henning, W. Kwon, J. Lazio, H. Linz, L.G. Mundy, A.I. Sargent, S. Storm, L. Testi, D.J. Wilner, Constraints on the Radial Variation of Grain Growth in the AS 209 Circumstellar Disk. Ap. J. Lett. 760, 17 (2012)

L.M. Pérez, C.J. Chandler, A. Isella, J.M. Carpenter, S.M. Andrews, N. Calvet, S.A. Corder, A.T. Deller, C.P. Dullemond, J.S. Greaves, R.J. Harris, T. Henning, W. Kwon, J. Lazio, H. Linz, L.G. Mundy, L. Ricci, A.I. Sargent, S. Storm, M. Tazzari, L. Testi, D.J. Wilner, Grain Growth in the Circumstellar Disks of the Young Stars CY Tau and DoAr 25. Ap. J. 813, 41 (2015)

D. Perez-Becker, E. Chiang, Surface Layer Accretion in Conventional and Transitional Disks Driven by Far-ultraviolet Ionization. Ap. J. 735, 8 (2011)

A.-M.A. Piso, K.I. Öberg, T. Birnstiel, R.A. Murray-Clay, C/O and Snowline Locations in Protoplanetary Disks: The Effect of Radial Drift and Viscous Gas Accretion. Ap. J. 815, 109 (2015)

C. Qi, K.I. Öberg, D.J. Wilner, P. D’Alessio, E. Bergin, S.M. Andrews, G.A. Blake, M.R. Hogerheijde, E.F. van Dishoeck, Imaging of the CO Snow Line in a Solar Nebula Analog. Science 341, 630-632 (2013)

G.G. Sacco, E. Flaccomio, I. Pascucci, F. Lahuis, B. Ercolano, J.H. Kastner, G. Micela, B. Stelzer, M. Sterzik, High-resolution Spectroscopy of Ne II Emission from Young Stellar 
Objects. Ap. J. 747, $142(2012)$

R. Salmeron, M. Wardle, Magnetorotational instability in stratified, weakly ionized accretion discs. MNRAS 345, 992-1008 (2003)

T. Sano, J.M. Stone, The Effect of the Hall Term on the Nonlinear Evolution of the Magnetorotational Instability. II. Saturation Level and Critical Magnetic Reynolds Number. Ap. J. 577, 534-553 (2002)

J.B. Simon, X.-N. Bai, J.M. Stone, P.J. Armitage, K. Beckwith, Turbulence in the Outer Regions of Protoplanetary Disks. I. Weak Accretion with No Vertical Magnetic Flux. Ap. J. 764, 66 (2013a)

J.B. Simon, X.-N. Bai, P.J. Armitage, J.M. Stone, K. Beckwith, Turbulence in the Outer Regions of Protoplanetary Disks. II. Strong Accretion Driven by a Vertical Magnetic Field. Ap. J. 775, 73 (2013b)

J.B. Simon, G. Lesur, M.W. Kunz, P.J. Armitage, Magnetically driven accretion in protoplanetary discs. MNRAS 454, 1117-1131 (2015)

D.R. Soderblom, L.A. Hillenbrand, R.D. Jeffries, E.E. Mamajek, T. Naylor, Ages of Young Stars. Protostars and Planets VI, 219-241 (2014)

D.J. Stevenson, J.I. Lunine, Rapid formation of Jupiter by diffuse redistribution of water vapor in the solar nebula. Icarus 75, 146-155 (1988)

M.H.R. Stoll, W. Kley, Particle dynamics in discs with turbulence generated by the vertical shear instability. Astron. Astrophys. 594, 57 (2016). doi:10.1051/0004-6361/201527716

T. Takeuchi, C.J. Clarke, D.N.C. Lin, The Differential Lifetimes of Protostellar Gas and Dust Disks. Ap. J. 627, 286-292 (2005)

M. Tazzari, L. Testi, B. Ercolano, A. Natta, A. Isella, C.J. Chandler, L.M. Pérez, S. Andrews, D.J. Wilner, L. Ricci, T. Henning, H. Linz, W. Kwon, S.A. Corder, C.P. Dullemond, J.M. Carpenter, A.I. Sargent, L. Mundy, S. Storm, N. Calvet, J.A. Greaves, J. Lazio, A.T. Deller, A multi-wavelength analysis for interferometric (sub-)mm observations of protoplanetary disks: radial constraints on the dust properties and the disk structure. ArXiv e-prints (2015)

N.J. Turner, S. Fromang, C. Gammie, H. Klahr, G. Lesur, M. Wardle, X.-N. Bai, Transport and Accretion in Planet-Forming Disks. Protostars and Planets VI, 411-432 (2014)

N. van der Marel, E.F. van Dishoeck, S. Bruderer, S.M. Andrews, K.M. Pontoppidan, G.J. Herczeg, T. van Kempen, A. Miotello, Resolved gas cavities in transitional disks inferred from CO isotopologs with ALMA. Astron. Astrophys. 585, 58 (2016)

K.J. Walsh, A. Morbidelli, S.N. Raymond, D.P. O'Brien, A.M. Mandell, A low mass for Mars from Jupiter's early gas-driven migration. Nature 475, 206-209 (2011)

M. Wardle, A. Königl, The structure of protostellar accretion disks and the origin of bipolar flows. Ap. J. 410, 218-238 (1993)

S.J. Weidenschilling, The distribution of mass in the planetary system and solar nebula. Astrophysics and Space Science 51, 153-158 (1977)

J.P. Williams, W.M.J. Best, A Parametric Modeling Approach to Measuring the Gas Masses of Circumstellar Disks. Ap. J. 788, 59 (2014)

R. Xu, X.-N. Bai, On the Grain-modified Magnetic Diffusivities in Protoplanetary Disks. Ap. J. 819, 68 (2016)

A.N. Youdin, Y. Lithwick, Particle stirring in turbulent gas disks: Including orbital oscillations. Icarus 192, 588-604 (2007) 